\title{
Historias paralelas: la fortaleza de Els Vilars y el oppidum de Puente Tablas
}

\section{Parallel histories: the Els Vilars Fortress and the Puente Tablas oppidum}

En este artículo se contrastan dos casos de asentamiento de la cultura ibera, que oponen dos modelos diferentes de patrones de asentamiento: uno micronuclear con poblamiento disperso en torno a Els Vilars de Arbeca (Lleida) y otro polinuclear con concentración absoluta de la población en Puente Tablas (Jaén). A partir de esta diferencia se propone una reflexión sobre los modelos políticos en la cultura de los iberos distinguiendo entre los diferentes tipos de príncipes, sus bases ideológicas y sociales y sus orígenes.

Palabras clave: cultura ibera, patrones de asentamiento, modelos sociales, Els Vilars, Puente Tablas.
This article contrasts two opposing settlement pattern models of the Iberian Culture. The first is micronuclear formed by a sparse settlement around Els Vilars de Arbeca (Lleida) while the other is polynuclear consisting of an absolute concentration of population in the sites of Puente Tablas (Jaén). The differences lead to a reflection on the political models of the Iberian Culture distinguishing between the different types of princes, their ideological and social bases and their origins.

Keywords: Iberian Culture, settlement patterns, social models, Els Vilars, Puente Tablas.
Era habitual que cuando terminaba la intervención, un arqueólogo, siempre el mismo, discutía mis propuestas, observando la debilidad de los datos con los que construía mis conclusiones. Defendía yo que la servidumbre territorial, base de nuestros debates, se fijaba tanto desde los datos empíricos, como desde la articulación dialéctica de estos con conceptos de la teoría marxista de la Historia. Era en los Coloquios de Historia Antigua que organizaba la Universidad de Oviedo en la segunda mitad del decenio de los setenta del siglo pasado y el arqueólogo se llamaba Emili Junyent y también pensaba desde el marxismo. De tanto debate sobre datos, método y conceptos nos hicimos amigos. Han pasado cuarenta años, yo completé parte de aquellos datos que no tenía, él hizo otro tanto en el territorio ilergete y ambos hemos madurado los viejos conceptos marxistas. Como en varias ocasiones Emili me ha pedido que valore sus propuestas, no sé si para compensar aquellos debates unidireccionales de los primeros encuentros, he pensado que este es el mejor lugar para recuperar una antigua tradición historiográfica, 
la comparación de dos modelos socioeconómicos, los territorios de la Cuenca del Cinca-Segre y del Alto Guadalquivir, dos casos, uno de iberos del norte y otro de iberos del sur. De este modo me sumo al homenaje que bien merece Emili Junyent por su constancia y rigor en el trabajo y por su coherencia de pensamiento. Hay algo falso en esta recuperación escenográfica, porque ninguno de los dos actores somos ya personas individuales, en realidad diría que en este momento identificamos un grupo amplio de investigadores que a lo largo de estos años se han sumado al trabajo y han caracterizado con su labor los resultados de la investigación, es decir que todos juntos conformamos un Emili Junyent y un Arturo Ruiz colectivos, discúlpeseme esta licencia que es meramente literaria.

Aunque algo intuíamos, Emili y yo, no teníamos, hace cuarenta años, la certeza absoluta de que estábamos afrontando dos casos muy diferentes de sociedades iberas. Diría hoy que los dos extremos del espectro sociopolítico de una amplia gama de modelos. En este largo proceso el monolitismo que caracterizaba la lectura de la cultura e historia de los iberos se ha derrumbado para dar paso a una variedad de situaciones, que, no obstante, mantienen puntos en común: el carácter aristocrático de la sociedad, su definición urbana, un sistema de relaciones sociales propias de un modelo tributario de economía y, por supuesto, que todas las sociedades iberas fomentaron el desarrollo de la desigualdad social y dieron paso a estructuras de estado para afirmarla. En el marco de esta nueva diversidad que se ha asentado al paso del tiempo retomemos en las siguientes líneas el estado del debate... cuarenta años después.

\section{Dos tramas urbanas diferentes en un espacio fortificado único}

La comparación de los modelos urbanos de $\mathrm{Pu}-$ ente Tablas en Jaén y Els Vilars en Arbeca, ambos de tamaño medio en el contexto regional de su territorio, nos ofrecen dos dibujos distintos de la concepción espacial del grupo social que residía en el asentamiento. No obstante esta diferencia general, ambas comunidades vivieron integradas en un espacio fortificado desde inicios del siglo VIII a.n.e. en Vilars y desde mitad del vII a.n.e. en Puente Tablas, casi dos siglos después de la fundación de un asentamiento de cabañas. No creo que fuera la temprana etapa de la fortificación del primero lo que condujo a diseñar un modelo de urbanismo de trama cerrada con espacio central, donde destacaba la existencia de un pozo-cisterna, que se mantuvo activo durante todo el tiempo de vida del asentamiento hasta fines del siglo IV a.n.e. (Junyent y López 2016). Se podría haber modificado el diseño espacial, si la estructura social lo hubiera requerido, quiere decir esto que hubo algo que lo hizo pertinente mientras estuvo activa la fortaleza. Del primer urbanismo del oppidum de Puente Tablas, tras la construcción de la fortificación, no sabemos apenas nada, salvo que al tiempo que se construyó la muralla torreada se abandonaron las cabañas y nació la casa de planta cuadrangular (Molinos y Ruiz 2015; Ruiz et al. 2015). A comienzos del siglo vi a.n.e., sin embargo, se construyó un trazado de manzanas, separadas por calles paralelas, que perduró hasta el abandono del sitio a fines del siglo IV a.n.e. (aunque después volvió a ocuparse en

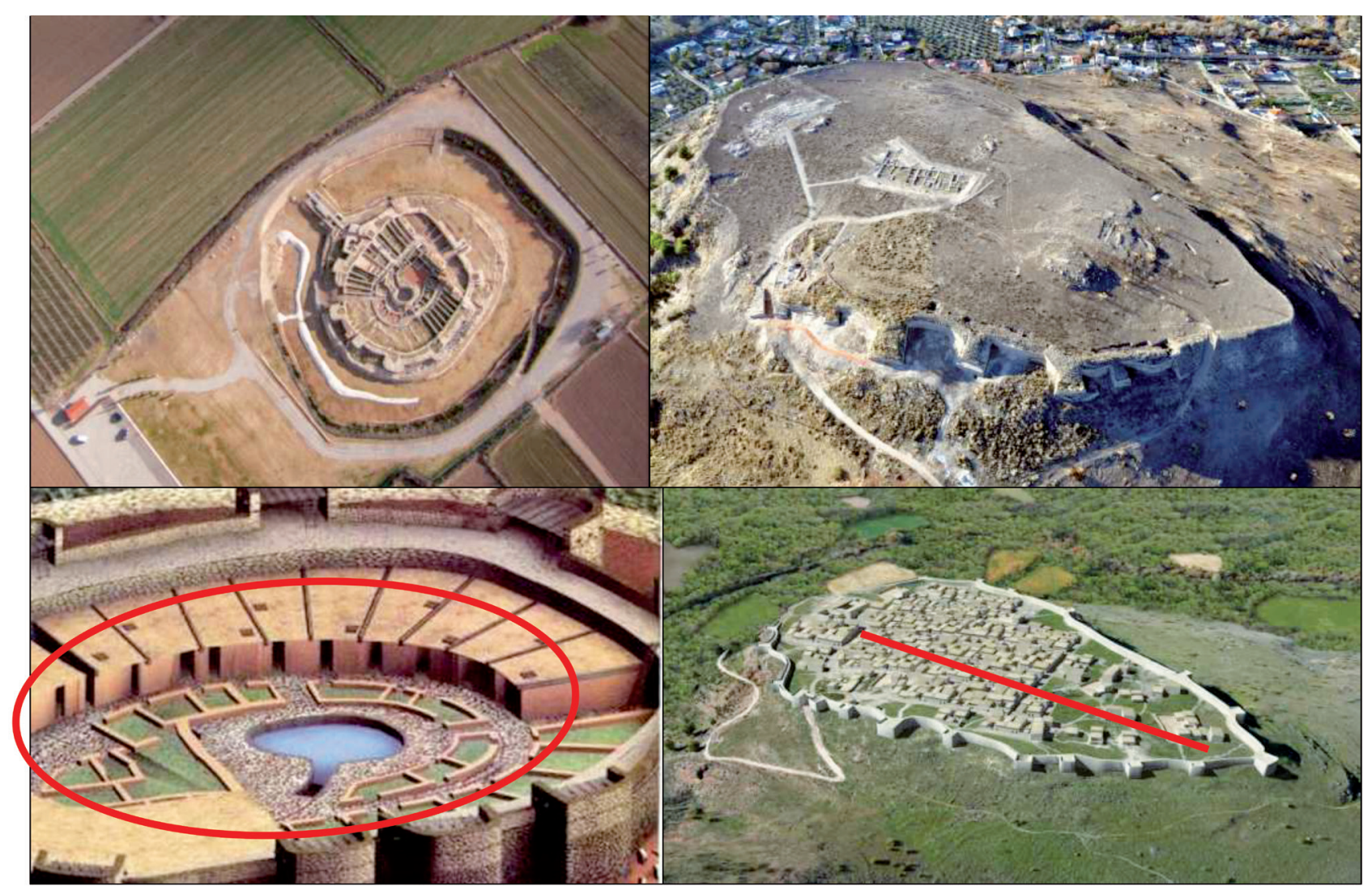

Figura 1. Modelos urbanos comparados de la fortaleza de Els Vilars de Arbeca y el oppidum de Puente Tablas de Jaén. 
la segunda mitad del siglo III a.n.e.). Apunto esta simultaneidad entre el primer abandono de Puente Tablas y el definitivo de Els Vilars, porque no creo que se tratara de una coincidencia a tenor de los datos que nos llegan de los modelos territoriales del siglo III a.n.e. Es un tema sobre el que volveré después. La estructura urbana circular de Els Vilars, frente a la longitudinal de Puente Tablas, refleja un modelo social diferente (figura 1). En el asentamiento ilergete priman los valores comunales, frente a las formas longitudinales jerarquizadas de las manzanas paralelas de Puente Tablas, lo que hace que se presenten las casas en condiciones de igualdad, en el que no destaca ninguna unidad, al menos hasta Vilars II. Es entonces cuando una de las estructuras radiales, al norte del círculo, se hace destacar en el conjunto de la trama urbana con la construcción de un espacio de mayor tamaño, que no tiene el carácter familiar de las demás casas y que parece asumir funciones comunales, con un hogar tipo lingote chipriota en el centro de la sala, la presencia de un altar, un betilo y un banco adosado a la pared del fondo. No cabe duda de que se trata de un espacio cultual, sin embargo no habría que descartar que representara además un lugar de prácticas comunales de cohesión política, estoy pensando en una sala de reunión para el grupo en armas al modo de las estructuras arquitectónicas que acogían la curia en el ámbito etrusco y romano. Llama la atención la casa que se dispone al oeste del espacio de culto, porque, no obstante desconocer sus características, al menos por tamaño y sobre todo por proximidad a la estructura anterior, podría constituir la residencia de una familia relativamente destacada de la comunidad. No hace falta que tenga mejor calidad de materiales muebles. En una estructura de base igualitaria como la que se lee en el urbanismo cerrado de Vilars, la jerarquía interna del grupo puede recurrir exclusivamente a factores de posición y tamaño. En el túmulo colectivo de Cerrillo Blanco del siglo vII a.n.e. en Porcuna, la disposición de la tumba más importante es excéntrica al resto del grupo y se separa de él por un área de respeto, por su carácter de enterramiento de pareja y, en este caso sí, por los materiales de construcción: grandes lajas de piedra que conforman una cámara poligonal enlosada; sin embargo, los materiales de prestigio que se han documentado: fíbula de doble resorte, cuchillos afalcatados de hierro, peine decorado o broches de cinturón, circulan por el resto de las tumbas y no se documentan en la citada cámara principal (Torrecillas 1985; Ruiz y Molinos 2015).

En el Puente Tablas del siglo vi a.n.e. el orden longitudinal no permitía sostener las pautas igualitarias de la distribución en círculo. El eje principal del urbanismo define en el centro de la meseta el núcleo del caserío, en manzanas y calles paralelas, y tras un espacio vacío hacia el sur, se levantan al menos dos edificios que caracterizan el área aristocrática, uno de los cuales pudo ser un palacio, sobre el que se construyó a mediados del siglo $\mathrm{v}$ a.n.e. otro que lo doblaba en tamaño. No obstante, en el siglo VI a.n.e. se han podido documentar dos lagares en una de las habitaciones, que realzan el papel aristocrático del espacio. En el otro extremo del eje, al norte, se documenta en los siglos V-IV a.n.e una gran cisterna que pudo estar activa en la fase anterior. Como se ve, son los mismos elementos que en Els Vilars, si bien siguiendo pautas espaciales diferenciadas: caserío circular en Arbeca que integra los espacios simbólicos-colectivos y seguramente los espacios de poder, desde luego no monumentalizados, y estructura comunal para el necesario recurso del agua ocupando el centro del círculo. Frente a ello Puente Tablas jerarquiza un eje longitudinal, presidido por el área de representación del poder en posición segregada y destacada del resto, como si se tratara de una casiacrópolis que se levanta en el extremo de la meseta, y que ya integra producciones no comunitarias como el vino; después el conjunto de manzanas con calles paralelas, que apuntan hacia el área aristocrática y caen desde el centro de la meseta hacia la base del espacio de poder, tras un gran espacio abierto sin tratamiento constructivo. Por último, detrás del caserío las infraestructuras comunales, aquí en la posición opuesta al ámbito privado aristocrático. Una diferencia importante a subrayar es el tema de los espacios vacíos o no ocupados al interior de la fortificación, que en el oppidum de Puente Tablas, en este momento del siglo vi a.n.e. es un espacio significativo en tamaño, comparado con el conjunto de las áreas construidas, lo que facilita el crecimiento demográfico del oppidum sin tener que modificar sus estructuras de defensa; frente a él el caso ibero del norte, Els Vilars, desde su construcción limita el crecimiento del grupo social que lo habita, salvo las modificaciones que posibilitan algunas reestructuraciones internas de las casas. En el primer caso el oppidum es un núcleo con crecimiento potencial, algo que forma parte de su nacimiento como unidad abierta a su desarrollo interno, en cambio Els Vilars es un micronúcleo, por su reducido tamaño y porque su estructura circular no permite crecimiento potencial.

\section{Caudillo, príncipe... y el caso del bigman}

La aristocracia está ligada históricamente al nacimiento del tributo y a las relaciones sociales que sostienen su existencia y que se fijan por formas de dependencia entre clases sociales; para definirla, propongo tres horizontes de análisis. El primer campo lo constituye la servidumbre como estructura básica de las relaciones sociales de producción. Se parte del hecho de que la sociedad ibera tiene su origen en modelos socio-económicos sostenidos por relaciones de parentesco desde las que se tiende a construir estructuras de desigualdad social. Esta variante permite concluir que existieron al menos tres formas de servidumbre según que el grupo dependiente y tributario se configurara en el seno de la propia estructura de parentesco, fuera de ella o articulando un modelo mixto. Partiendo de este factor, origen interno o externo de los grupos dependientes respecto a la estructura de parentesco, la primera forma de servidumbre se reconoce en la estructura del clan cónico, que es una construcción social jerarquizada, surgida en el seno del grupo de parentesco, en función de la posición de proximidad parental de cada linaje 
a los antepasados fundadores del clan; la segunda forma de servidumbre parte, al contrario que la anterior, de un grupo tributario que no pertenece a la estructura de parentesco o que, formando parte de ella, cambió la naturaleza de sus relaciones, el caso más frecuente es la servidumbre por rendición tras un conflicto, aunque pueden existir otras formas. En este caso el grupo dependiente nunca forma parte de la estructura de parentesco del grupo dominante, ni siquiera en términos de parentesco ficticio, y se expresa normalmente a través de la servidumbre territorial a partir de un pacto (deditio). Torelli la define como un tipo de clientela (Torelli 1996), sin embargo en la historiografía española hay una tradición en el uso de la servidumbre territorial que creo interesante mantener. En alguna ocasión este tipo de servidumbre deriva en el que veremos a continuación: la clientela gentilicia, así sucede con Anco Marcio en Roma, cuando traslada a la ciudad a los vencidos por deditio y los reparte entre las novae curiae, convirtiendo la servidumbre territorial en clientela gentilicia (Torelli 1996), siempre con la condición previa de integrar estos grupos en el núcleo urbano. La tercera y última forma de servidumbre es precisamente la clientela, y parte de un grupo externo que a través de un pacto in fides entre cliente y patrono (applicatio), se integra en el núcleo urbano de este. En el caso de la clientela gentilicia el grupo se incorpora además a la estructura de parentesco de modo ficticio, como si realmente tuviera lazos de sangre, y con la condición de desplazar al ámbito privado su genealogía, una especie de amnesia pública de los antepasados propios. Para que esta clientela se desarrolle hace falta un ajuste en las relaciones entre el núcleo familiar y el linaje de tal modo que se ha de producir un pacto político entre el oikos, que toma ahora la forma abierta de la casa, y el linaje, que debe definirse como gens (Ruiz y Molinos 2018). En todo caso esta clientela puede partir o ser coetánea de un modelo que Torelli define como clientela regia (Torelli 1996) y que aquí la caracterizaremos como clientela patriarcal. En este caso se trata de la creación de una servidumbre que no se integra en el linaje por parentesco, aunque realiza el pacto de fidelidad y se incorpora al núcleo urbano del patrono, en condición de individuos libres, igual que la anterior, de ahí el carácter suave de la clientela como forma de dependencia. La diferencia entre uno y otro tipo de clientela reside en que en el caso de la última, la patriarcal, su naturaleza es pública, en tanto que en la clientela gentilicia es privada por el pacto entre oikos y linaje gentilicio. Una última forma de clientela se hace patente en un marco histórico diferente, me refiero a las relaciones interaristocráticas, en las que al pacto in fides se une las estrategias de alianza matrimonial, como se hace patente en la boda de Aníbal con la princesa de Castulo, Himilce, o en el pacto de fidelidad de Indíbil con Escipión (Ruiz y Molinos 2018). La característica de esta clientela es que se expresa en más de un rango de dependencia, uno urbano que se identifica con las formas nucleadas de clientela ya expuestas y otro que se sitúa por encima de ellas y que definiremos como clientela interaristocrática, territorial y con estructura piramidal. Sabemos que esta última clientela tiene su expresión desarrollada en la devotio (Ramos y Loscertales 1924; Prieto 1978). Las tres formas de servidumbre, incluidos los tres tipos de clientela citados, no son excluyentes entre sí (en el caso de la clientela piramidal es obligado que conviva con cualquiera de las otras dos) y todas pueden interactuar en el seno de una única formación social. Lo importante es determinar cuál de las tres formas de servidumbre y en su caso de clientela es dominante en la estructura de las relaciones sociales.

El segundo horizonte a valorar para definir la estructura política y social de los iberos, parte de la relación creada entre las dos instituciones que caracterizan las relaciones de parentesco (sean estas reales o ficticias): el linaje (no necesariamente gentilicio) y la familia (el oikos). Para el caso de la clientela gentilicia las dos instituciones adquieren una nueva expresión social debido a la capacidad que tienen para integrar de modo ficticio grupos externos, hablo del linaje cuando alcanza la forma de gens y sustituye el linaje de parentesco real y del oikos abierto en oposición a la familia nuclear u oikos cerrado y desde luego hablo de su compleja articulación (Ruiz y Molinos 2018). En el primer caso, la gens o linaje gentilicio ha sido bien definida a partir de la arqueología italiana, en los trabajos de Torelli (Torelli 1996), Peroni (Peroni 2004) o Carandini (Carandini 2014), en todo caso destaco como posicionamiento teórico que ya no se trata del tradicional linaje de parentesco. El caso del oikos abierto a la integración de personas externas a la familia, tiene una de sus mejores lecturas conceptuales en los trabajos de Lévi-Strauss sobre las sociedades de "Maison", cuando al definirlas escribe: "La maison est d'abord une personne morale, détentrice ensuite d'un domaine composé de biens matériels et immatériels. Par immatériel, j'entends ce qui relève des traditions, par matériel, la possession d'un domaine réel ... Ainsi que je l'avais indiqué, c'est à ce type d'unité qu'il y a quelques années j'ai proposé d'appliquer le terme de maison. Celle-ci se perpétue en transmettant son nom, sa fortune et ses titres en ligne directe ou fictive tenue pour légitime à la seule condition que cette continuité puisse s'exprimer dans le langage de la parenté ou de l'alliance et le plus souvent des deux ensemble" (Lamaison y Lévi-Strauss 1987: respuestas 2 y 4).

Un tercer horizonte, por último, lo define el concepto de residencia, tanto en los espacios de la vida como en los espacios de la muerte. En uno de los extremos del sistema espacial, estaría la servidumbre territorial en la que los dos grupos sociales, dependientes y dominantes, suelen mantener sus residencias originales separadas; en cambio, la clientela gentilicia, en el otro extremo, tiende a incorporarse a los espacios del grupo nucleado, espacios de residencia del patrono en la vida y en la muerte, seguramente para consolidar la ruptura de los lazos con su genealogía original al incorporarse políticamente a otro linaje, ahora gentilicio, y/o al oikos. Así se observa en el paisaje funerario de la necrópolis de Baza, en la que arqueológicamente se distingue el área de la clientela del de la parentela y se observa la disposición de los enterramientos de los clientes en las proximidades de la tumba 176, del príncipe y su familia, mientras que 
los de la parentela se aproximan al enterramiento n. ${ }^{\circ} 155$ de la Dama de Baza, la generación fundadora de linaje gentilicio (Ruiz y Molinos 2018). Es evidente que el uso del mismo espacio para realizar la deposición de los restos funerarios de ambos grupos es indicativo de que existe una alianza entre el oikos y la gens para caracterizar el espacio de la muerte. Se trata de una expresión espacial a la que la interpretación arqueológica ha dado visibilidad, pero no quiere decir que tenga que leerse de la misma manera que en Baza en todas las necrópolis iberas conocidas. En Puente Tablas, a mediados del siglo v a.n.e., se reforma la fortificación, se construye el gran palacio sobre el anterior y la plaza existente entre el palacio y las manzanas del siglo vi a.n.e. se ocupa con casas, observándose un significativo crecimiento demográfico, que podría justificar la incorporación de la clientela. Este proceso no se puede desarrollar en las fases ibéricas de Els Vilars, porque la estructura urbana no lo permite, pero es posible que sí comenzara a hacerse realidad en casos como Molí de l'Espigol de Tornabous, con la reestructuración de la primera fortificación (Principal et al. 2012) a partir de fines del siglo $\mathrm{v}$ a.n.e.

En relación directa con este horizonte ha de valorarse el modelo de asentamiento que define el espacio de residencia de la aristocracia y sus unidades de parentesco ficticio o real. Me refiero al triple modelo: celular, micronuclear y nuclear (Ruiz 2008). Parece evidente que los dos primeros difícilmente pueden sostener el paradigma residencial de la clientela observado en Puente Tablas, tal y como se advertía para el caso de Els Vilars, lo que refuerza la idea que en los dos primeros modelos era difícil que se desarrollara la forma de la clientela, aunque sí era posible la servidumbre territorial y desde luego el clan cónico. El reciente mapa del territorio de Els Vilars con la estructura de polígonos de Thiessen entre fortalezas del mismo tipo y con la existencia en cada polígono de asentamientos no fortificados (Junyent y López 2016) podría ser indicativo de que entre los centros de poder y las aldeas existía uno de los dos primeros tipos de servidumbre citados, el clan cónico o la servidumbre territorial. Será la investigación arqueológica en los próximos años la que tendrá que fijar si estos asentamientos formaban parte de la estructura de parentesco o no. En caso de ser un clan cónico debió estar muy jerarquizado internamente.

Dialécticamente el modo de producción, es decir las relaciones de tributación y sus formas de servidumbre articulados al modo de vida que definen las relaciones de parentesco y alianza y las relaciones de residencia, dibujan distintos tipos de aristocracia. Príncipe es el concepto que yo he utilizado habitualmente, siguiendo la tradición académica franco-italiana (Torelli 1996; VV AA 1999; Ruiz 1998 y 2000), Caudillaje, hasta al menos el siglo III a.n.e. es el término empleado por E. Junyent para los aristócratas que gobernaban las fortalezas tipo Els Vilars (Junyent 2015; Junyent y López 2016). No se trata de utilizar aleatoriamente, según distintas tradiciones académicas, unas u otras categorías, el pensamiento no es inocente. Creo que podría ser válida la articulación de las tres variantes

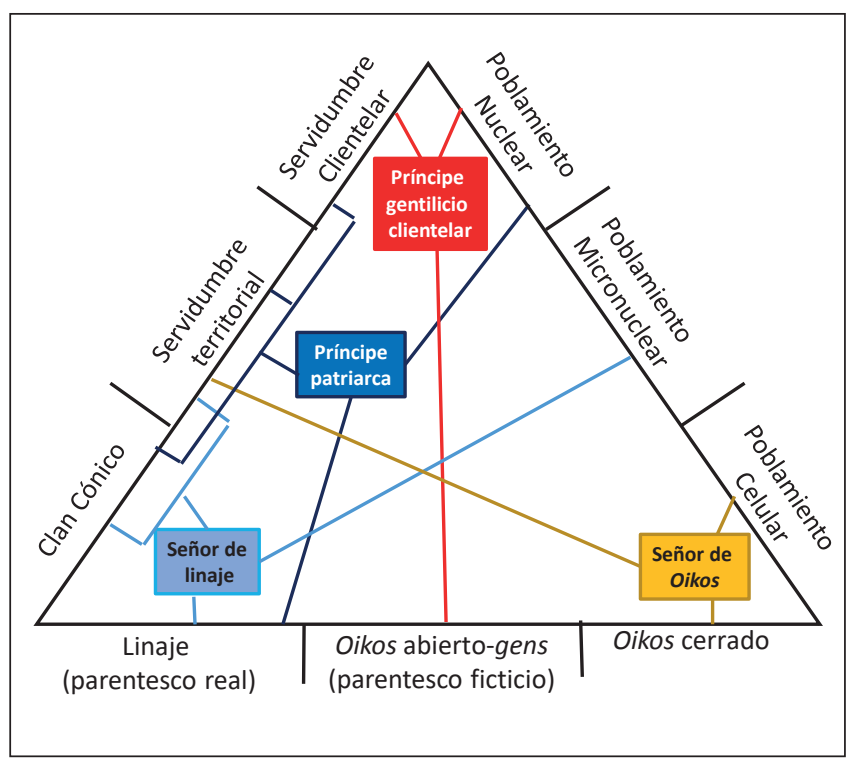

Figura 2. Tipología de las aristocracias iberas según la triple variante establecida (siglos vII-IV a.n.e.).

valoradas hasta aquí para fijar las categorías que definen el poder en la aristocracia ibera, es decir para cargar de conceptos más precisos los términos empleados (Figura 2). Por caracterizar los dos casos citados: el caudillo de la fortaleza ilergete se define en un modelo de asentamiento micronuclear como residencia de un linaje poco jerarquizado internamente y con las unidades familiares diluidas en la trama urbana, en plano de igualdad. Este tipo de residencia del poder se articula en el marco de una comunidad más amplia y dispersa, localizada al exterior de la fortaleza y cuya relación de dependencia se expresa o por la forma de la servidumbre territorial o por un clan cónico con una estructura social muy jerarquizada entre los linajes del clan. Por el contrario, un príncipe del Alto Guadalquivir de los siglos V-IV a.n.e. se expresa en un modelo de asentamiento con nucleación absoluta, en el que está segregado gracias a su estructura longitudinal, el espacio del poder del oikos, si bien todo el asentamiento se configura como la residencia de un linaje gentilicio, tal y como se observa en Puente Tablas o a veces compartiéndolo con otros linajes gentilicios como en Cástulo. Puede resolverse esta diferencia, en términos arqueológicos, por dos vías, una por el tamaño del oppidum, pues en su escala mayor, cuando alcanza cifras superiores a las diez hectáreas, parece aceptable que convivieran varias gens, tal y como se deduce de los conflictos internos de Castulo antes de entregarse a los romanos en el 206 a.n.e.; y, de otra parte, por el número de necrópolis coetáneas, cada una de las cuales podría representar un linaje distinto.

Entre estos dos modelos existen significativas variantes. En el mundo de los príncipes pueden establecerse hasta dos situaciones diferentes, dos tipos distintos, partiendo siempre de que la nucleación es una invariante de su condición de existencia y de que los oikos aristocráticos se segregan del resto de la comunidad dentro del área nucleada, ubicándose en un espacio 
privado, que en los casos más jerarquizados toma la forma de un complejo palacial y en los menos de una o varias residencias, como expresión de varios oikos compitiendo entre sí (Grau y Vives-Ferrandiz 2018). Sin embargo, hay una notable diferencia entre la institución de la que arranca la desigualdad y el tipo de clientela desarrollada. El príncipe gentilicio clientelar que se dibuja conceptualmente, en Puente Tablas a partir de la segunda mitad del siglo v a.n.e. es el fruto de una aristocracia que configuró sus espacios en el siglo vi a.n.e., quizás porque ya existía este tipo de clientela; sin embargo, en los espacios de la muerte no llegaron a desarrollarse las formas espaciales características del linaje gentilicio clientelar hasta más tarde, tal y como se observa en Baza. Estos príncipes protogentilicios, en ocasiones, hombres como en Pozo Moro o mujeres como en la necrópolis del Santuario de Baza y las más parejas como en Cabezo Lucero y seguramente en la tumba de Cástulo estudiada por Blanco (Blanco 1965; Ruiz y Molinos 2018), marcaron el lugar de partida del paisaje funerario donde después se desarrollarían las grandes necrópolis clientelares, pero no fueron ellos los que construyeron el espacio gentilicio de la muerte, ni siquiera incorporando a este espacio la parentela con la que tenían relaciones de parentesco real. Su ideal del espacio funerario es la tumba, más o menos monumentalizada, aislada y a lo sumo familiar. Sin embargo, como se ha hecho notar anteriormente, se trata de príncipes reconocibles arqueológicamente por sus espacios de residencia y que construyeron los oppida con la estructura urbana jerarquizada de espacios organicistas tan queridos a los príncipes gentilicios clientelares. En todo caso, y si, como seguramente ocurrió, tuvieron pequeñas clientelas (porque el sistema gentilicio no nace por decreto), desde luego no se visibilizaban ni en los espacios de la muerte, ni claramente en los espacios de la vida. Ahora bien, son príncipes que segregaron los espacios de residencia del oikos aristocrático y que nuclearon sus linajes en el oppidum. Además, la pervivencia de un segundo nivel de asentamientos de tipo aldea en el siglo vi a.n.e. reafirma que su clientela pudo no ser todavía dominante frente a otras formas de servidumbre como la territorial. Si esta referencia a los enterramientos origen de las necrópolis clientelares se observa en toda el área oretano-bastetana, en cambio en el área turdula de fuerte tradición tartésica, donde dominaron las necrópolis tumulares, unas veces en túmulo colectivo (Cerrillo Blanco) y otras con túmulos individuales asociados en un mismo espacio (La Noria, Fuente Piedra, Málaga) (Ruiz et al. 2017), reivindican un modelo de origen muy distinto al anterior, al diluirse el oikos en el espacio funerario en favor del linaje de parentesco real (debo decir que no lo tengo tan claro para el caso del túmulo de Cerrillo Blanco). Los reconocemos como príncipes patriarcas, pues aunque parten de principios semejantes a los de los caudillos, por la vigencia de las relaciones de parentesco, muestran un grado de jerarquía mayor, desde luego en el espacio funerario del linaje por los materiales constructivos, por el uso de mayor variedad de maderas en la pira por las ofrendas en el foso del túmulo y por la apropiación de objetos de prestigio aristocrático en el ajuar, como en La Noria, Fuente de Piedra, los equipos de libación, compuestos por jarra y brasero (Ruiz et al. 2017); sin embargo, el colectivo funerario participa en La Noria y en Cerrillo Blanco de la circulación de objetos de producción externa y alto valor económico. Llama también la atención que tanto en La Noria como en Cerrillo Blanco los enterramientos no tienen armas, un hecho que contrasta con los anteriores enterramientos de los príncipes de oikos, a los que sí se les deponen estas en el ajuar de sus tumbas y además no solo armas ofensivas, sino también defensivas. En lo demás su capacidad para aglutinar la población en el oppidum no deja dudas sobre su potencial identidad como príncipes, como también la dificultad que implicaría con estos modelos conservar durante mucho tiempo una estructura de parentesco real, en un marco tendente a aumentar la jerarquía. De hecho hay datos significativos que hacen dudar de la viabilidad del modelo y de su supervivencia en el Alto Guadalquivir, pues ciento cincuenta años después del cierre del túmulo de Cerrillo Blanco, se construyó el monumento escultórico de Porcuna, que es imagen ya de los príncipes gentilicios posteriores. Es posible que durante el siglo vi a.n.e. se produjera una fuerte iberización de la zona, como en algún momento ha apuntado de Hoz (De Hoz 2015), que diera al traste con el modelo de príncipe patriarcal, si es que llegó a existir, permitiendo que el sistema oikosgens recondujera también allí la expresión espacial de las nuevas necrópolis tal y como parecen confirmar algunas tumbas documentadas en el Fondo Marsal, lamentablemente objeto de expolio, de necrópolis de la Campiña de Jaén como los Chorrillo de iliturgi en Mengibar o la Carada en Espeluy (Catálogo), sin olvidar otras necrópolis excavadas en la década de los cincuenta como la de La Eras de San Sebastián en la Guardia de Jaén (Blanco 1959). El hecho coincide con la colonización advertida a fines del siglo VII a.n.e., y que apenas alcanzó el siglo vi a.n.e., en los ríos situados en el límite de las provincias de Jaén y Córdoba, Salado de Porcuna y Guadatín, desarrollada por oppida como Torreparedones en Baena, Montoro o Ipolca en Porcuna al facilitar una ocupación por pequeñas factorías agrarias, residencias seguramente de unidades familiares jerarquizadas en al menos dos rangos (uno de los cuales se presentaba como centro de producción metalúrgica, alfarera y centro de distribución, caso de Las Calañas en Marmolejo), que podrían justificar la vigencia de unidades de linaje suprafamiliar (Molinos et al. 1994). El proyecto se abortó en su expansión cuando el pagus del arroyo de Arjona o de Los Villares fortificó en los inicios del siglo vi a.n.e. su territorio con torres de tipo Coronilla de Cazalilla evidenciando que ya existían en ese momento territorios con oppida jerarquizados en la Campiña de Jaén. En este caso con el oppidum de Villargordo en el centro del Valle apoyado en cuatro pequeños núcleos: Atalayuelas de Fuerte del Rey, con una pequeña y potente fortificación, Torrejón, Torrebenzala y Cerro Miguelico. La coincidencia entre la desaparición de los asentamientos familiares de Ipolca y la amortización definitiva del túmulo de Cerrillo Blanco con su modelo de patriarca, podría anunciar 
el principio del fin del modelo (no tengo claro que el patriarca se pueda definir como príncipe en el área túrdula, aunque no cabe duda que existieron príncipes patriarcas en el área tartesia central del Bajo Guadalquivir) de linaje de parentesco real en la Campiña de Jaén y su sustitución por los príncipes de los oikos, que registramos en la construcción a fines del siglo VI a.n.e. de las tumbas fundadoras de las necrópolis clientelares de fines del siglo v a.n.e. de la Campiña de Jaén. Este proceso en cambio no se advierte en estas fechas en la necrópolis de La Noria, en La Hoya de Antequera, que continuará reproduciendo las necrópolis de linaje, al menos hasta inicios del siglo $\mathrm{v}$, aunque con túmulos individualizados y jeraquizada al menos en tres rangos (posiblemente cuatro).

Por resumir la cuestión, en el Alto Guadalquivir se parte de dos modelos políticos opuestos en el siglo vII: el patriarca en la Campiña de Jaén y Córdoba y el príncipe de oikos en la parte oriental de Jaén, como punta de iceberg de un amplio territorio que se extendía por Albacete, Alicante, Murcia o Granada. Ambos tenían sus mejores y antagónicas expresiones funerarias en el túmulo colectivo de Cerrillo Blanco y en la tumba de Cástulo de las Hathor (Blanco 1965). Hacia mitad del siglo vi a.n.e. el modelo de patriarca desapareció sustituido por los príncipes de oikos o protogentilicios. Fue entonces cuando estos construyeron un pacto institucional entre la unidad familiar del oikos abierto y la conversión del linaje en gens, que dará lugar a los príncipes gentilicios clientelares que se harán visibles arqueológicamente a fines del siglo $\mathrm{v}$ y sobre todo durante el siglo iv a.n.e.

En el caso del modelo ilergete y sus caudillajes, he de confesar que como categoría que identifique este tipo de aristocracia me parece más ajustado el término "señor", tal y como se ha empleado en recientes trabajos sobre las aristocracias rurales de la Extremadura postartésica, que residen en asentamientos celulares o edificios señoriales como en la Mata o Cancho Ruano y que muestran en su entorno pequeños asentamientos rurales sin duda dependientes de cada unidad señorial (Rodríguez 2009). No se trata del mismo caso de Els Vilars de Arbeca, que, como he tratado de mostrar siguiendo a Junyent, son micronúcleos que definen la residencia de un linaje, pero la escala de poder me parece equiparable y el conflicto entre unidad familiar y linaje que se ha observado en el Sur y que es lo que me interesa destacar, está también aquí presente. No obstante, para llegar a ello es obligado ampliar la escala territorial hasta alcanzar el Bajo Ebro, y valorar un caso que ha contado en los últimos años con un amplio tratamiento historiográfico y un debate de gran interés. Se trata de las casas-torre (Moret 2001; Moret et al. 2006) y antes de modelos como el Complejo Sant Jaume (García 2001 y 2015), que responden a residencias celulares del poder como en Extremadura.

En los últimos años se ha debatido si estos personajes que se segregan de la comunidad en el Bajo Ebro responden a la figura del bigman (Sanmartí 2010 y 2015) o a un modelo de jefatura simple (García 2015). Efectivamente, coincido con las críticas que García expresa sobre la posibilidad de identificar la familia de la residencia aristocrática de Sant Jaume con la familia de un bigman. Como bien destaca este autor, esta última figura se inscribe en un sistema político-económico igualitario regido socialmente por reglas de parentesco. Su excepcionalidad se expresa en que por primera vez se hace visible una estrategia capaz de poner en cuestión el modelo de reciprocidad don-contradón al controlar el bigman los circuitos de distribución de determinados productos de prestigio y desarrollar formas de don agonístico a través de grandes ceremonias como el potlatch; sin embargo, este nivel de competencia entre bigman que rompe las normas de reciprocidad y endeudan simbólicamente a quienes se benefician de la posesión y consumo de los productos controlados por él, en ningún momento pone en crisis el sistema de parentesco ya que la intervención económica del bigman no llega a generar una estructura tributaria y formas de dependencia más allá del reconocimiento a su prestigio. De hecho, su debilidad es tal que es frecuente en periodos de crisis su reintegración al núcleo comunal, porque no ha perdido su lugar en las relaciones de parentesco que rigen el sistema social. En todo caso, que el aristócrata de Sant Jaume difícilmente se pueda identificar con un bigman, no quiere decir que la figura de este no sea clave para entender el proceso que dio origen al CSJ. Para que surja un bigman son necesarias tres cuestiones, en opinión de Godelier (Godelier 1998): en primer lugar que el matrimonio se produzca por dote y no por intercambio de mujeres; en segundo lugar que haya competencia en la acumulación y redistribución de riquezas, y en tercer lugar que entren bienes de prestigio en la circulación de dones además de bienes de subsistencia. Las tres cuestiones son fundamentales en el origen de la aristocracia y en el desarrollo de formas de desigualdad y yo añadiría otra cuarta cuestión, que los bigman siempre se muestran en unidades familiares y no arrastran en su aventura a los linajes. En mi opinión el bigman es un ensayo desde la sociedad igualitaria de modelos prearistocráticos con base en el oikos. Fueron estos hechos los que Godelier consideró de interés cuando en el congreso de Barcelona presentó la figura del bigman a los iberistas, sin ser consciente de que descontextualizado y sin valorar las relaciones de producción en que nacía, este personaje podía correr por los tiempos iberos sustituyendo otras formas de representación aristocrática. Con todo, los fundamentos que dan lugar al nacimiento del bigman son sin duda principios fundamentales en la definición de las aristocracias de base familiar o de oikos.

De los cuatro factores señalados anteriormente, salvo el primero, la dote (o los hedna tan habituales en la sociedad homérica de la Ilíada) exige un análisis profundo y contrastado de los espacios domésticos y funerarios. Los otros tres: control de la circulación de los productos de prestigio fenicios, la competencia aristocrática, que se lee en el claro control del territorio de Sant Jaume, incluidas las complejas defensas de la residencia, además de las prácticas de comensalidad y fundamentalmente de consumo del vino y la segregación de la residencia del resto de la comunidad que estaría representada en La Moleta, no dejan dudas de que los principios del bigman habían sido asimilados en la estrategia de la residencia aristocrática de Sant 
Jaume. Falta sin embargo por analizar un factor fundamental que excluiría definitivamente la identidad del señor de Sant Jaume con el bigman y caracterizaría las nuevas estructuras como aristocráticas y que expreso a través de una pregunta que no he observado en los debates. ¿Fue capaz Sant Jaume de crear un sistema de relaciones tributarias, aunque este fuera de naturaleza muy básica? En mi opinión los tres asentamientos: La Ferradura, el Castell d'Ulldecona y la Cogula, que configuran el control del territorio del Complejo, deberían ser preguntados por las razones que hicieron que sus habitantes abandonaran el espacio colectivo de la comunidad para constituir una red de servicios en favor de Sant Jaume. En ello se debió basar la dependencia entre estos grupos y la residencia aristocrática y son ellos fundamentalmente quienes hicieron visible la servidumbre en el modelo político, que les alejaba del bigman.

El CSJ no duró mucho tiempo. Tampoco tuvieron un largo recorrido las casas-torre, que pudieron haber representado la generalización posterior del modelo en el territorio. La propuesta había fracasado cuando La Moleta se recuperó sin perder su carácter comunal de poblado cerrado con espacio central abierto. La experiencia de los señores de oikos había terminado con claridad en el siglo v a.n.e. cuando entraba en un momento de máxima monumentalidad la fortaleza de Els Vilars. Al contrario que en el Alto Guadalquivir, la estructura política de residencia de los señores del linaje se había impuesto en el Valle del Ebro sobre la de los señores del oikos. La doble vía había abierto dos caminos que confluirían sin embargo a fines del siglo IV a.n.e. cuando tanto Els Vilars como Puente Tablas fueron abandonados.

\section{Príncipes de príncipes. La realeza ibera}

La desaparición de la fortaleza de Els Vilars y del oppidum de Puente Tablas a fines del siglo IV a.n.e. (aunque este último volvió a ser ocupado a fines del siglo III a.n.e. en el marco de la Segunda Guerra Púnica, para desaparecer definitivamente algunos años después), no es un hecho casual. Los dos abandonos son fruto de un cambio político que derivó en el siglo III a.n.e. en la formación de los estados territoriales que conocemos en su fase final a través de Polibio y Tito Livio. Son consecuencia por ello de un ajuste político interno en el marco de la maduración de los procesos aristocráticos iberos y responden sin duda a los ajustes internos que dieron lugar en ambos territorios al Estado Arcaico. En ese proceso en la Cuenca del Cinca-Segre nacieron posiblemente los príncipes, sin que necesariamente desaparecieran todos los señores. Molí de l'Espígol es un buen ejemplo de la evolución de las fortalezas, pues ya desde fines del siglo $v$ a.n.e., pero sobre todo a fines del siglo IV a.n.e., se producen dos hechos que hacen que la fortaleza se nuclee y se convierta en un auténtico oppidum: con el primero la fortificación más antigua se amortiza y nace una segunda línea de muralla al exterior de la primera, que permite ampliar el espacio de hábitat, alcanzando la hectárea de tamaño, e incorporar nuevos grupos sociales (Principal et al.
2012). El segundo hecho desde el punto de vista urbano es también importante, pues aunque, también en la nueva fortificación, se mantiene en general la tradición radial de la disposición de las casas familiares, sin embargo el espacio central del oppidum, símbolo comunal en las fortalezas de tipo Els Vilars, ahora se ocupa con edificios privados entre los que destaca la residencia de un príncipe (Monrós 2010) con un tamaño superior al de las demás casas y con dos columnas en la entrada del edificio que dignifican y legitiman a sus residentes. La naturaleza social de los nuevos grupos integrados en el oppidum no está definida, pero en razón al proceso que hemos seguido hasta aquí, se ha de pensar que se incorporan a través de alguna forma de dependencia clientelar. Seguramente son los viejos linajes del clan cónico o de la posible servidumbre territorial que rodeaban las fortalezas, los que ahora se muestran como clientela, que no necesariamente tiene que abrir los linajes a relaciones gentilicias, sino que podrían expresar algún tipo de características semejantes a la clientela patriarcal, no gentilicia, aunque desde luego partiendo del pacto in fides. Este modo abre el tercer tipo de clientela, la piramidal con carácter territorial, cuya existencia mostró Indíbil al convertirse en cliente de Escipión y que tuvo su expresión más desarrollada con la devotio, pero existió también con respecto a la dependencia de Indíbil de los príncipes lacetanos o ausetanos. En todo caso no dejaron de existir los poblados cerrados con su área central no ocupada o reiterando fórmulas como la cisterna, como se observa en Estinclells (Asensio et al. 2016) en el siglo III a.n.e., aunque, ahora sí, es posible observar, en la todavía reiterada disposición radial, diferencias entre las casas, hasta distinguir por tamaño y distribución interior tres grupos jerarquizados, dispuestos por zonas.

La reocupación de Puente Tablas es realmente de gran interés y no es una anécdota, porque los nuevos habitantes del oppidum de fines del siglo III a.n.e. parecen ser los descendientes del viejo linaje gentilicio del siglo Iv a.n.e. pues vuelven a ocupar las mismas casas conservando el trazado longitudinal de las manzanas y las calles paralelas; reocupan también el palacio, sin apenas modificaciones estructurales significativas, ya que conservan el gran patio central con columnas de madera sobre basas de piedra, aunque recuperan y modifican el espacio de culto a los antepasados, cerrándolo y colocando un betilo a la entrada, con orientación astronómica al ocaso del equinoccio. En el conjunto urbano, hay sin embargo cambios importantes, pues significativamente se amortiza y olvida, cubriéndolo ritualmente con tierra cribada, el santuario de la Puerta del Sol, abandonando definitivamente el espacio de culto, y se modifica significativamente la monumentalidad de la Puerta del Sol de la fortificación hasta convertirla en un pequeño portillo, al tiempo que rehacen la fortificación ya sedimentada, con pequeños torreones. Parece como si se ritualizara en esta parte del asentamiento una damnatio memoriae contra los cultos del siglos v-IV a.n.e. ¿Dónde han estado estas gentes durante casi un siglo, habiendo mantenido activa la estructura de su linaje gentilicio? ¿Qué les ha llevado a volver a la antigua residencia gentilicia? Seguramente no son 
ajenos a la decisión las acciones políticas y militares de la Segunda Guerra Púnica y que el final del oppidum se deba a un asedio romano a fines del siglo III o inicios del siglo II a.n.e. (Lechuga et al. en prensa).

La construcción de los estados arcaicos a fines del siglo IV o inicios del III a.n.e. ha sido objeto de diferentes trabajos (Ruiz 1998; Santos 1998; Junyent 2015) y también se han hecho notar las diferencias entre el territorio ilergete y el oretano-bastetano, entre un estado constituido por unidades de oppida en el sur y otro por unidades étnicas o populi en el norte (Ruiz 1998; Coll y Garcés 1998). No voy a reiterar el tema, sí añadir y hacer notar para los iberos del sur un trabajo nuevo que ha permitido dibujar arqueológicamente la estructura territorial de uno de estos estados: el de Castulo. Me refiero al estudio de la distribución de los exvotos de bronce de la colección Marsal que hasta hace poco, solamente se habían encontrado en los dos grandes santuarios territoriales en Cueva de Sierra Morena: Collado de los Jardines en Despeñaperros y Cueva de la Lobera de Castellar, y que hemos vinculado tradicionalmente con el pagus de Cástulo (Ruiz y Molinos 2007). La colección de exvotos de Marsal (Ruiz y Rueda 2014), aunque procedente de expolio, tiene referencias de los sitios donde los bronces fueron hallados y ello ha permitido visualizar una red de siete oppida (Cástulo, Giribaile en Vilchez, Espeluy, Turruñuelos (Baecula), Úbeda la Vieja (Iltiraka), Loma del Perro en Jodar y seguramente Tugia) que se articulan alrededor de Cástulo y de sus santuarios, la gran capital que emitió moneda a fines del siglo III a.n.e. A ello se ha unido recientemente el hallazgo de un tercer santuario, el Haza del Rayo en Sabiote, en este caso localizado en la Loma de Úbeda, junto a un posible pequeño lago, en un paso tradicional que cruzaría del Valle del Guadalquivir al del Guadalimar (excavación inédita dirigida por C. Rueda) y que articularía territorialmente los oppida de los dos valles.

Príncipes de príncipes del sur, que al alcanzar dos rangos o más de clientela cabría definir como reyes (figura 3), que mantienen vivas sus estructuras gentilicias y que se jerarquizan entre sí seguramente a partir de pactos in fides y alianzas matrimoniales, lo que daría lugar a clientelas interaristocráticas piramidales no gentilicias y territoriales (Ruiz y Molinos 2007), de ahí la variabilidad de los dominios de Culchas entre el 206 y el 198 a.n.e. de 28 a 17 oppida. También príncipes de príncipes, reyes del norte, que construyen oppida (no muchos, solamente dos en el territorio ilergete - Junyent 2015), nuclean sus clientelas, no necesariamente gentilicias, como parece proponer la ausencia de necrópolis en este periodo, y que, al contrario que los príncipes del sur, parecen mantener vigente en el territorio la estructura de linajes de parentesco, sobre todo en las escalas superiores de las entidades étnicas. Príncipes estos en los que la vigencia de la estructura en populi les facilita la concreción territorial de la clientela piramidal y con ello del estado arcaico sobre la base de la confederación intertribal, como muestra la asociación jerarquizada de ilergetes, ausetanos y lacetanos bajo el control militar de Indíbil.

Reyes de ambos territorios, que vieron como caían sus prístinos estados a partir de las revueltas de 198197 a.n.e. (de los siete oppida de Castulo del siglo III a.n.e solo pervivirán dos, Castulo y Úbeda la Vieja. Posiblemente tres si se añade Tugia y de los dos

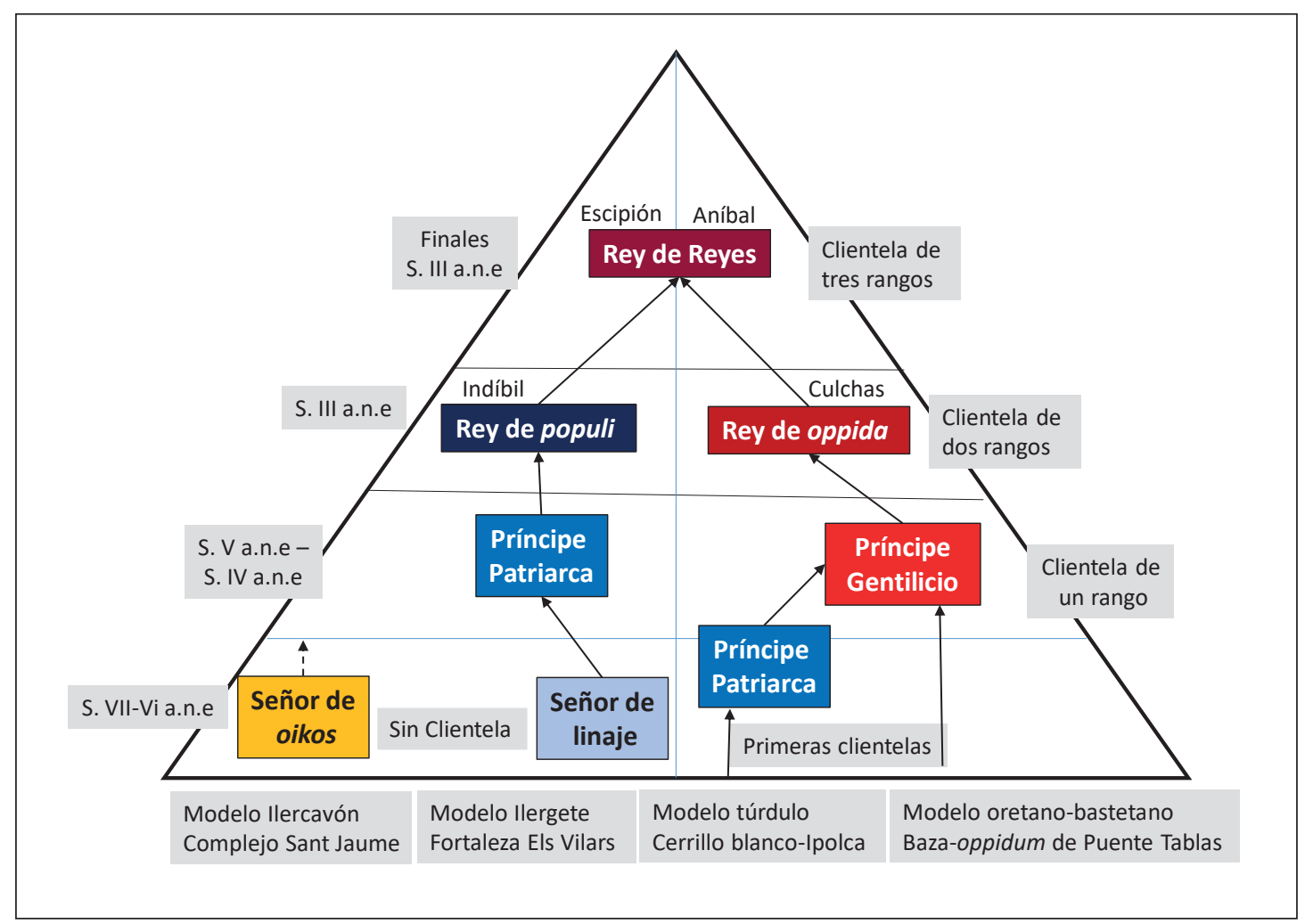

Figura 3. Evolución de las aristocracias iberas (siglos vII-I a.n.e.). 
oppida ilergetes, Tornabous fue destruido). Estados decapitados de sus cabeceras políticas, por la muerte violenta de sus reyes: Indíbil y seguramente Culchas, y como contrapeso un fortalecimiento de los viejos linajes prerromanos que al menos en el sur se refundaron sobre la memoria de las antiguas gens como muestra la presencia de keimelias, como las cráteras griegas de fines del siglo $\mathrm{v}$ e inicios del IV a.n.e. y las armas del siglo iv (una lanza y dos puñales de antenas recortadas) en el ajuar de la primera mitad del siglo I a.n.e. del príncipe, Iltirtiiltir, en Piquia, Arjona, que sin embargo se enterró a la romana, con gladius y lorica hamata (Ruiz et al. 2015)

Como se habrá observado por todo lo escrito hasta ahora, se trata de dos modelos, el ilergete y el oretano-bastetano, muy distintos en sus bases fundacionales, en las características básicas de sus geografías, en sus estructuras de poblamiento y en las formas de poder que los caracterizan, además de desarrollar diferentes procesos en la articulación de los tipos de servidumbre que definen sus relaciones sociales de producción. Sin embargo, si abstraemos los conceptos en una escala conceptual más amplia, ambos territorios siguen un proceso histórico común, que podría definirse ibero, y que aúna territorios tan diferentes como los comparados. Se trata de un proceso en cuatro fases: una primera en la que se configuran las aristocracias locales con la construcción de las fortificaciones, que no solamente son estructuras de defensa, sino también definidoras de la estructura interna del grupo dominante. Esta fase tiene distintas fechas de arranque pero se configuran claramente a lo largo del siglo vi a.n.e. La segunda etapa se desarrolla desde fines del vi y durante el siglo IV a.n.e. y define, sobre todo en este último siglo, la consolidación de los distintos modelos aristocráticos locales. Se caracteriza arqueológicamente por las huellas de la monumentalización, como la fortificación o el pozo-cisterna de Els Vilars o la remodelación de sitios como Molí de l’Espígol y en Puente Tablas es el momento de las reformas de la fortificación con la espectacular Puerta del Sol y sus ritos astronómicos de fertilidad, la construcción del nuevo palacio y sobre todo la definición de los nuevos espacios funerarios aristocráticos de las necrópolis con esculturas. La tercera fase transcurre desde fines del siglo IV y ocupa todo el siglo III a.n.e. reestructurando el territorio con las clientelas piramidales para dar lugar a los estados arcaicos desde diferentes modelos de organización política, lo que conlleva crisis y desaparición de algunas fortalezas y oppida y paralelamente la creación de capitalidades. Por último, tras la conquista romana se produce de forma generalizada la decapitación de los estados al tiempo que renacen refundados al menos en Andalucía los viejos linajes locales.
Arturo Ruiz Instituto Universitario de Investigación en Arqueología Ibérica Universidad de Jaén Paraje Las Lagunillas s/n, Jaén arruiz@ujaen.es 
Asensio, D., Cardona, R. Morer, J., Pou, J., Saula, O., Alonso, N. (2016). El jaciment dels Estinclells (Verdú, Urgell), una fortalesa ilergeta del segle III aC. Nova recerca a partir del Camp d'Experimentació de la Protohistòria (CEP). Tribuna de Arqueologia, 20132014: 152-177.

Blanco, A. (1959). Excavaciones arqueológicas en la provincia de Jaén. Boletín del Instituto de Estudios Giennenses, 6: 89-128.

Blanco, A. (1965). El ajuar de una tumba de Cástulo. Oretania, 19: 7-60.

CARANDini, A. (2014). La fundación de Roma. Bellaterra. Barcelona.

Coll, N., Garcés, I. (1998). Los últimos príncipes de Occidente: Soberanos ibéricos frente a Cartagineses y Romanos. En: C. Aranegui (ed.). Congreso Internacional Los Iberos Príncipes de Occidente (Barcelona, marzo, 1998). Barcelona.

GARcía, D. (2011). Nuevas aportaciones al estudio de los patrones de asentamiento en el nordeste de la Península Ibérica durante la Primera Edad del Hierro. El caso del Complejo Sant Jaume. Trabajos de Prehistoria, 68(2): 331-352.

GARcía, D. (2015). Jefes del Sénia. Sobre la emergencia de jefaturas durante la primera Edad del Hierro en el nordeste de la Península Ibérica. Munibe, 66: 223-243.

Godelier, M. (1998). Funciones, formas y figuras del poder político. Saguntum: Papeles del Laboratorio de Arqueología de Valencia, No Extra 1, 1998 (Ejemplar dedicado a: Actas del Congreso Internacional "Los Iberos, Príncipes de Occidente”, Centro Cultural de la Fundación "la Caixa"): 13-24.

Grau, I., J. Vives-Ferrandiz. (2018). Entre casas y comunidades: Formas de organización y relación social en el área oriental de la península ibérica (siglos v-II A.N.E.). En: A. Rodríguez, I. Pavón. D. Duque (eds.). Más allá de las casas. Familias, linajes y comunidades en la protohistoria peninsular. Universidad de Extremadura. Cáceres: 73-110.

Hoz, J. de (2015). La lengua ibérica en Jaén, desde el s. Iv hasta las inscripciones de Piquía y las Atalayuelas. Jaén, Tierra Ibera. Universidad de Jaén: 397-411.

Junyent, E., J. B. Lopez (2016). La Fortalesa dels Vilars d'Arbeca. Museo de Lleida. Lleida.

JunYENT, E. (2015). L'evidència arqueològica en la definició de la societat estatal arcaica ilergeta. En: Belarte, M. C., Garcia, D., Sanmartí, J. (eds.). Les estructures socials protohistòriques a la Gàl.lia i Ibèria. VII Reunió Internacional d'Arqueologia de Calafell. Arqueomediterrània: 14, 165-191.
Lamaison, P. y Lévi-Strauss, C. (1987). La notion de maison. Entretien avec Claude Lévi-Strauss par Pierre Lamaison. Terrain, octubre 1987: 34-39 < http://journals. openedition.org/terrain/3184> 2 y 4 .

Lechuga, M. A., Molinos, M., Ruiz, A. Bellon, J. P., Gómez, F., Valderrama, J. (en prensa). E la guerra giunse fin nelle nostre case...Tracce di un attacco alla porta nord dell'oppidum di Puente Tablas (Jaén). Edizioni Quasar.

Molinos, M., A. Ruiz (2015). La Fortificación del oppidum de Puente Tablas. En: Jaén, Tierra Ibera. Cuarenta años de investigación y transferencia. Universidad de Jaén. Jaén.

Molinos, M., J. L. Serrano, C. Risouez y S. Montilla (1994). Las Calañas de Marmolejo: Un problema de fronteras en la periferia de Tartessos. Universidad de Jaén. Jaén.

Monrós, M. (2010). L’Edifici Singular A de la ciutat ibèrica del Molí d'Espígol (Tornabous, l’Urgell): interpretació i funcionalitat. Cypsela, 18: 213-225.

Moret, P. (2001). El Tossal Montañés (Valdeltormo, Teruel): une maison-tour ibérique du vi siècle av. J.-C. Madrider Mitteilungen, 42: 85-104.

Moret, P., Benavente, J. A., Gorges, A. (2006). Iberos del Matarraña: investigaciones arqueológicas en Valdeltormo, Calaceite, Cretas y La Fresneda (Teruel). Al-QANNIS, 11.

Peroni, R. (2004). Culti, comunità tribali e gentilizie, caste guerriere e figure di eroi e principi nel secondo millennio in. Italia tra Europa centrale ed Egeo. En: F. Marzatico y P. Gleirscher (eds.). Guerrieri, Principi ed Eroi, fra el Danuvio e il Po, dalla Preistoria all'Alto Medievo. Catalogo. Trento: 161-173.

Principal, J., Asensio, D., SALA, R. (2012). L'espai suburbà de la ciutat ilergeta del Molí d'Espígol (Tornabous, l'Urgell). En: El paisatge periurbà a la Mediterrània occidental durant la protohistòria i l'antiguitat: actes del col.loqui internacional. Institut Català d'Arqueologia Clàssica. Tarragona: 165-182.

Prieto, A., (1978). La devotio ibérica como forma de dependencia en la Hispania romana. Memoria de Historia Antigua, 2: 137-146

Ramos y Loscertales, J. M. (1924). La “devotio ibérica”. Anuario de Historia del Derecho Español, 1: 7-26.

Rodríguez, A. (2009). Campesinos y "señores del campo". Tierra y poder en la protohistoria extremeña. Editorial Bellaterra. col. Arqueología. Barcelona.

Ruiz, A. (1998). Los príncipes Iberos. Procesos económicos y sociales. En: C. Aranegui (ed.). Congreso Internacional Los Iberos Príncipes de Occidente (Barcelona, marzo, 1998). Barcelona: 285-300. 
Ruiz A. (2000). El concepto de clientela en la sociedad de los príncipes. Saguntum-PLAPÏ Extra, 3: 11-20.

Ruiz, A. (2008). Íberos. En: Gracia Alonso, F. (coord.). De Iberia a Hispania. Ariel. Madrid: 733-844

Ruiz, A. y M. Molinos (2007). Iberos en Jaén. Universidad de Jaén. Jaén.

Ruiz, A., M. Molinos (2015). El conjunto escultórico de Cerrillo Blanco, Porcuna. En: Jaén, Tierra Ibera. Cuarenta años de investigación y transferencia. Universidad de Jaén. Jaén.

Ruiz, A., M. Molinos (2018). Genealogía, matrimonio y residencia en el proceso político de los iberos de Alto Guadalquivi. En: A. Rodríguez, I. Pavón, D. Duque (eds.). Más allá de las casas. Familias, linajes y comunidades en la protohistoria peninsular. Universidad de Extremadura: 41-72.

Ruiz, A., Molinos, M., Pérez, F., Montes, E., Ortuño, E. (2017). El túmulo C de la necrópolis de la Noria (Fuente de Piedra). ¿La tumba de las dos mujeres? En: A. Ruiz y M. Molinos (eds.). Catálogo de la Exposición La Dama, el Príncipe, el héroe y la Diosa. Junta de Andalucía: 119-123.

Ruiz, A., Molinos, M., Risouez, C., Gómez, F., Lechuga, M. A. (2015): La cámara de Piquía, Arjona. En: Jaén, Tierra Ibera. Universidad de Jaén: 357-374.

Ruiz, A., M. Molinos, C. Rueda, R. Fernández (2015). El palacio y el urbanismo del oppidum de Puente
Tablas, Jaén. En: Jaén, Tierra Ibera. Cuarenta años de investigación y transferencia. Universidad de Jaén: 108-120.

Ruiz, A., RuEdA, C. (2014). Los exvotos en bronce del Farmm: oppida y santuarios. En: Farmm fondo arqueológico Ricardo Marsal Monzón. Junta de Andalucía. Sevilla: 131-144.

SAnMartí, J. (2010). Demografía y cambio sociocultural: el caso de la Iberia septentrional. Arqueología Espacial, 28: 91-108.

SAnMartí, J. (2015). Long-Term Social Change in Iron Age Northern Iberia (ca. 700-200 BC). En: A. Bernard Knapp y P. van Dommelen (eds.). The Cambridge Prehistory of the Bronze and Iron Age Mediterranean. Cambridge University Press: 454-487.

Santos, J. (1998). Los iberos: Entre la consolidación de las elites y el surgimiento del Estado. En: C. AranEgui (ed.). Congreso Internacional Los Iberos Príncipes de Occidente (Barcelona, marzo, 1998). Barcelona: 339-404.

Torelli, M. (1996): Historia de los Etruscos. Editorial Crítica. Barcelona.

Torrecillas, J. F. (1985). La necrópolis de época tartésica de Cerrillo Blanco. Instituto de Estudios Giennenses. Jaén.

VV. AA. (1999). Les princes de la protohistoire et l'émergence de l'état. Centre Jean Bérard. Naples; Ecole Française de Rome. Roma. 